\title{
Wireless Sensors self-location in an Indoor WLAN environment
}

\author{
Miguel Garcia ${ }^{1}$, Carlos Martinez ${ }^{2}$, Jesus Tomas ${ }^{3}$, Jaime Lloret ${ }^{4}$ \\ Department of Communications, Polytechnic University of Valencia \\ 1'migarpi@teleco.upv.es, ${ }^{2}$ carmargo@epsg.upv.es, ${ }^{3}$ jtomas@dcom.upv.es,.jlloret@dcom.upv.es
}

\begin{abstract}
Sometimes wireless sensors could be placed randomly without predetermined coordinates, so systems to self-locate in an area should be studied. This paper shows two approaches developed by us where wireless sensors could find their position using WLAN technology. The scenario is an indoor environment that contains walls, interferences, multipath effect, humidity and temperature variations, etc., and both approaches are based on the Received Signal Strength Indicator (RSSI). The first approach uses a training session and the position is based on Neuronal Networks using the training measurements. The second approach uses triangulation model with some fixed access points, but taking into account wall losses. In both systems we have considered the variations measured to obtain a bigger accuracy in the sensor localization.
\end{abstract}

\section{Introduction}

Positioning systems are becoming so important in our lives because they make life easier and more comfortable (e.g. GPS is actually used in four out of ten cars). The main advantage of GPS system is that obtains the position getting signals only from the satellites, but its disadvantage is that the receiver has to be in line of sight from the satellite, which is a problem for indoor applications. Moreover, GPS system has very low precision. Satellite-based positioning systems are not suitable for indoor areas because of the lack of coverage and costs. For these reasons, it is preferable the use of wireless local area networks (WLANs) to deploy an indoor positioning system.

Several indoor position systems using different technologies exists (e.g. IEEE $802.11 \mathrm{~b} / \mathrm{g}$ [1], Bluetooth [2], RFID [3], Zigbee [4] y UWB [5]). In this article we will show an indoor position location based approach for sensors using IEEE $802.11 \mathrm{~b} / \mathrm{g}$ because this type of technology has the following main advantages in front of others:
- It permits crossing walls, so the system could be used in more than one room.

- It is easy and quick to install.

- The costs of IEEE $802.11 \mathrm{~b} / \mathrm{g}$ devices are lower than those of other technologies.

- WiFi can be used in indoor or outdoor places.

- It supports changes in the physical topology of the network.

- WiFi systems allow having a high number of users, so they are more scalable.

We can find three ways for knowing the location of users: a) triangulation method that requires at least three different estimates of the distance of the sensor from known fixed places (usually Access Points), b) using the direction or angle of arrival (AOA) of two distinct signals from known locations at least, and c) employing fingerprinting schemes (neuronal networks) where the receiver must do previous training.

There are two types of WLAN location determination systems: client-based and infrastructure based. The client-based systems use the signal strength models to build the profile of the site. Using measurements from visible access points (APs), and for location estimation, a client reports back signal strength measurements from them, and these measurements are compared against the signal strength model to locate it. On the order hand, infrastructurebased system that mixes the information received by the signal strength checked in a client with the Round Trip Time given by the test and probe messages sent by the access points to the clients using tags.

In our analysis we have developed and tested two systems. One is based on the triangulation method and other that is based on a heuristic method using neuronal networks. We think that these two positioning systems are the ones that better adapt to wireless sensor networks because the processing data work made by the sensor is small, so wireless sensors will consume less energy. In addition, these location systems will be helpful to detect sensors in real environments where the node position changes constantly. 
The paper is organized as follows. Section 2 presents the technological background. Section 3 describes what types of Wireless Channel Variations are given in a WLAN environment. Section 4 provides two Indoor Location Systems developed by us. The performance evaluation and the comparative of both systems are shown in section 5 . Section 6 concludes the paper.

\section{Previous works}

In this section, we will relate several known indoor positioning systems, and describe their most important characteristics. It will give the lecturer the state of the art related with our system and our study.

The earliest location system was Active Badge [6] which is based on the use of badges worn by the users that emit infrared signals. The badge communicates about every 10 seconds a unique identifier that is picked up by fixed receivers. This is sent to a central server that provides an API. The accuracy of location depends on the number of receivers. To get very good locations it is needed many receivers. The Active Bat location system [7] uses a combination of radio signals and ultrasound. Periodically a base station transmits a radio message containing a single identifier, that it is referred with a device called a bat. Using the speed of the sound in the air, the times of flight of the ultrasound pulse from the Bat to the receivers can be converted into corresponding Bat-Receiver distances. If distances from the Bat to three or more non-collinear receivers can be found, its position may be determined. In this location system the precision can become to 9 $\mathrm{cm}$.

On the other hand, the Cricket location system [8] uses beacons that concurrently send information its location over RF, together with an ultrasound pulse. The receiver uses the time difference between the receipt of the first bit of RF information and the ultrasonic signal to determine the distance to the beacon. If a listener receives signals from at least three beacons, it is able to determine its location. The RADAR position system [9] is based on IEEE 802.11 wireless networks. This system uses received signal strength (RSS) from multiple access points (APs) positioned to provide overlapped radio map in the area of interest. In the online location phase, it combines empirical measurements with signal propagation modelling to determine the user location and thereby enables location aware services and applications. So, RADAR system requires that a mobile user must be able to see three or more APs to finish the location service. The more APs the user can see the more precision locations the system can reach. The median resolution of the RADAR system is in the range of 2 to 3 meters, about the size of a typical local area. Ekahau [10] provide location systems based on a similar approach used in RADAR. Ekahau uses the power vector method to calculate the user's position starting from the AP's power. The training process of the devices is carried out measuring the signals that are received from AP's in some calibrating points. The calculation of the position vectors is carried out in different way depending on if the device is active (PDA, computer) or passive (tags). In the estimate process, for each localization point the AP's signal intensity is compared with the database created in training process in order to determine the user's position. Ekahau's patented positioning technology features up to 1 meter average accuracy, enabling people and asset tracking both indoors and outdoors.

The Nibble location system, from UCLA, uses a Bayesian network to infer a user location [11]. Their Bayesian network model includes nodes for location, noise, and access points (sensors). The noise signal ratio observed from an access point at a given location is taken as an indication of that location. The system also quantizes the SNR into four levels: high, medium, low, and none. The system stores the joint distribution between all the random variables of the system.

Herecast [12] is another system based on WLAN. This system creates a database where the information for each AP is stored. Every time we come closer to an unknown AP we have to save their data in the database referring the data with the AP's position. In the localization process, as we get near to a known AP we can obtain our position referred to the AP position stored in the database. Herecast recognizes a near access point, and using a heuristic method, the wireless device can locate its position.

It is very difficult to increase the accuracy of location for those systems which use RSS or SNR as the principal system of measurement. The main reason is that these signals from APs are dominated by reflections, diffractions, and scattering of radio waves within an indoor environment.

Actually, the Horus location system [13] is the only one that uses received signal variation in their calculations. This system uses the signal strength observed for frames transmitted by the access points to infer the user location. When keeping in mind this parameter the Horus system identifies different causes for the wireless channel variations and addresses them to achieve its high accuracy.

In our triangulation method we have introduced the multi-path effect and the wall losses (none of the systems aforementioned had taken them into account). 
On the other hand, in our heuristic method we have introduced a signal correction based on the received signal variation and every time the sensor estimates its position, we can tell it its deviation from the correct place, so it is always training.

\section{Wireless Channel Variation}

In this section we are going to study the oscillation of received signal. We will try to introduce smaller degree errors in the location service.

In [14] we can see the three main variation caused in the RSS:

- Temporal variations: when the receiver remains in a fixed position, the signal level measure varies as time goes on.

- Small-Scale variations: the signal level changes when the device is moving in small distances, below the wavelength. For networks $802.11 \mathrm{~b} / \mathrm{g}$ working at $2.4 \mathrm{GHz}$ the wavelength is $12.5 \mathrm{~cm}$.

- Large-Scale variations: the signal level varies with the distance due to the attenuation that the RF signal suffers with the distance. Also known as multipath effect.

Besides these typical variations of the RF signal joined with the receiver mobility, we have also considered the temperature and humidity variations, the effect of opening and closing doors, the changes in the localization of the furniture, and the presence and movement of human beings, which are characteristics of indoor environments.

In the next subsections we will analyze the main RSS variations in a scenario where all the previous characteristics exist. We have chosen an university floor where there are several laboratories, corridors and offices. Measurements have been taken in school days where all the conditions are given.

\subsection{Temporal Variations}

It is a variation that takes place in the received signal along the time in a fixed position. In order to estimate it, we have used four APs and we have taken samples every minute during a period of 24 hours for two different distances. The floor distribution and the location of the APs to take measurements could be seen in figure 1 . The floor has $29.68 \times 75.47$ meters.

In figures 2 and 3, we can check the signal variation where there are four APs. We can observe that there are several moments with higher signal strength variations. It is given because there were people moving in the room. Signals received from AP2 and AP4 have higher variations from the mean value because they are placed in corridors where there use to walk many people. These APs are also the more influence APs in the Room 1. We can check that in the room 2 , it only happens with the signal received by the AP1.

\subsection{Small-Scale Variations}

Small-scale variations appear when there are small movements of the sensor. To study these effects we have measured movements of few centimetres from the receiver regarding a reference position, taking samples in each one of these positions. These movements, always below the signal wavelength $(12.5 \mathrm{~cm})$, have been carried out on a straight line and we have taken over 80 samples in each position with the purpose of obtaining a representative histogram and filtering the instantaneous noise of the measurement. Measurements have been taken at the reference point $(0 \lambda)$, at $3 \mathrm{~cm}$ from the reference point $(1 / 4 \lambda)$, at $6 \mathrm{~cm}$ from the reference point $(1 / 2 \lambda)$, at $9 \mathrm{~cm}$ from the reference point $(3 / 4 \lambda)$ and at $12.5 \mathrm{~cm}$ from the reference point $(\lambda)$.

The main variation is given over $1 / 2 \lambda$ by the more influence Access Point, the closest AP. We can check it in figure 4. We can also observe that the signal variation does not follow a regular law, but it seems to be random. It is the same conclusion that paper with reference [14] achieves.

\subsection{Large-Scale Variations}

Large-scale variations are the ones that are related with the distance because of the RF signal attenuation with regard to the distance. To check this effect we have divided the floor in cells separated 1 meter. The experiment consists on observing the changes of the received signal strength.

As we have done in previous subsections, in order to have a more representative histogram, and with smaller instantaneous noise, we have taken 70 samples in each place.

In figure 5, we can check a RSS variation with regards with the distance from the AP. There is higher signal strength when the distance from the sensor to the AP is lower.

Now we can state that the measured RSS is not well related with the distance from the AP for indoor environments. Indoor position models should take into account environment characteristics such as walls, interferences, multipath effect, humidity and temperature variations, and those given because of people walking, so they must be introduced. 


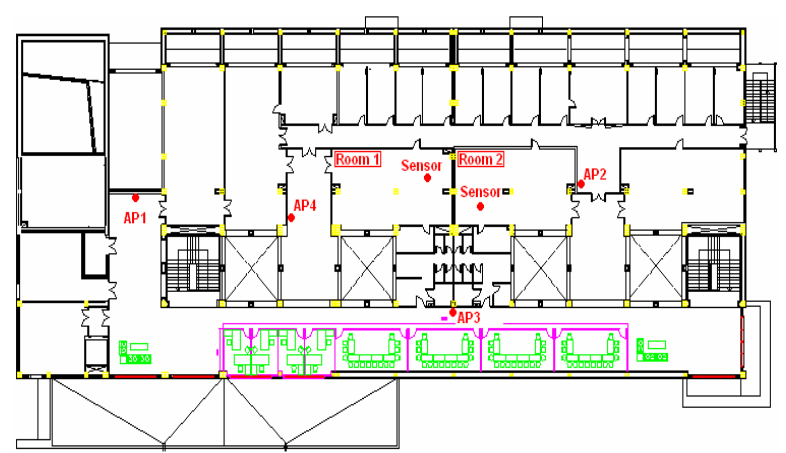

Figure 1. Testbed Floor and locations of the APs.

Time (hours)

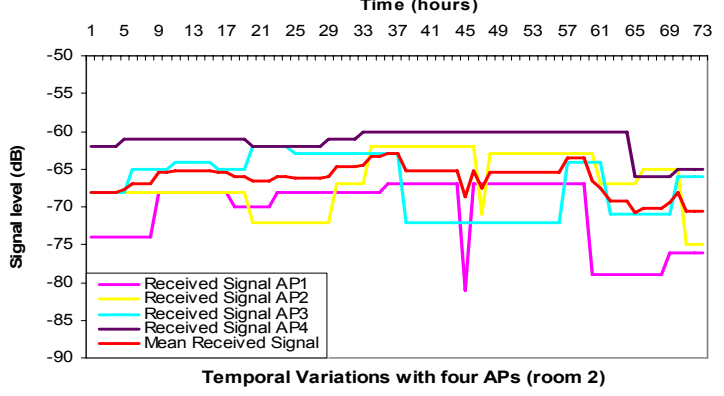

Figure 3. Temporal Variation in room 2

Time ( $\mathrm{min})$

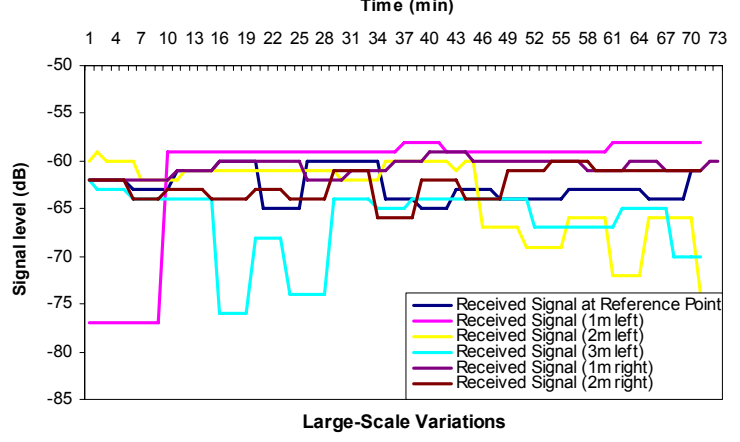

Figure 5. Large-Scale Variations.

\section{Indoor Location Systems}

We have developed two Indoor Location systems. One of them is based on triangulation system and the other is based on a Heuristic system. We have introduced several parameters such as walls effect, interferences, multipath effect, humidity and temperature variations, to obtain more accuracy measurements.

\subsection{Neuronal Networks Based System}

This system should be divided in two processes:

- Training process.

- Localization process.

In the training process, we use a reference map of the receive signal strength from many points of the place where the indoor localization system will work.

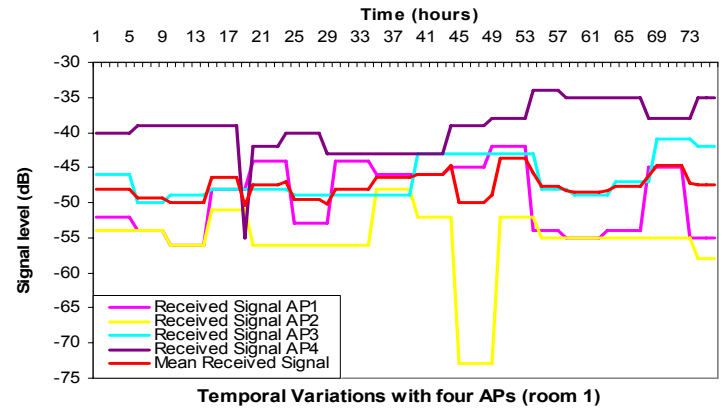

Figure 2. Temporal Variation in room 1 Time $(\mathrm{m}$ in)

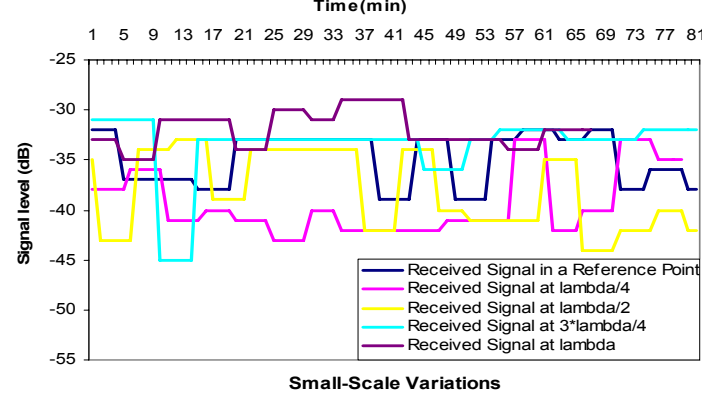

Figure 4. Small-Scale Variations.

Moreover, as we have seen in the previous section, the wireless signal has many variations in indoor environments, so, during this process, we have also taken into account the variations shown previously in order to obtain a better precision at the localization process. The measurements are stored in a database. An example given for the database is shown in table 1. The application saves, for each training point, the receive signal strength for each AP in our environment. The database will have an AP for each column, and as rows the number of positions stored during the training process.

Once we have completed the training process, we can start the localization process. The method selected to calculate the position is an inductive method. With this method expressions are defined based in the observed behaviour of every case. The position is obtained using comparisons between the received signal strengths and the stored values at the database during the training map, but taking into account the temporal variations, small-scale variations and the large-scale variations. Comparisons are taken using formulas given in figure 6. $a, b, c$ and $d$ are the receive signal strengths in the place where the sensor is located and $a_{n}, b_{n}, c_{n}$ and $d_{n}$ are the reference levels stored in the database. Each value obtained is associated to a reference position (e.g. $\mathrm{z}_{0}$ is associated to $\left(\mathrm{x}_{0}, \mathrm{y}_{0}\right)$ position). With all $z_{\mathrm{n}}$ values, we can use equation 1 to determine le location where the sensor is placed. Minimum values are used to reduce the error of $\mathrm{z}_{\mathrm{n}}$. 


\begin{tabular}{|l|l|l|l|l|}
\hline Position & $\mathbf{A P}_{\mathbf{1}}$ & $\mathbf{A P}_{\mathbf{2}}$ & $\mathbf{A P}_{\mathbf{3}}$ & $\mathbf{A P}_{\mathbf{4}}$ \\
\hline$\left(\mathrm{x}_{0}, \mathrm{y}_{0}\right)$ & $\mathrm{a}_{0}$ & $\mathrm{~b}_{0}$ & $\mathrm{c}_{0}$ & $\mathrm{~d}_{0}$ \\
\hline$\left(\mathrm{x}_{1}, \mathrm{y}_{1}\right)$ & $\mathrm{a}_{1}$ & $\mathrm{~b}_{1}$ & $\mathrm{c}_{1}$ & $\mathrm{~d}_{1}$ \\
\hline$\left(\mathrm{x}_{2}, \mathrm{y}_{2}\right)$ & $\mathrm{a}_{2}$ & $\mathrm{~b}_{2}$ & $\mathrm{c}_{2}$ & $\mathrm{~d}_{2}$ \\
\hline$\left(\mathrm{x}_{3}, \mathrm{y}_{3}\right)$ & $\mathrm{a}_{3}$ & $\mathrm{~b}_{3}$ & $\mathrm{c}_{3}$ & $\mathrm{~d}_{3}$ \\
\hline
\end{tabular}

Table 1. Database table.

$$
\begin{aligned}
& z_{0}=\sqrt{\left(a-a_{0}\right)^{2}+\left(b-b_{0}\right)^{2}+\left(c-c_{0}\right)^{2}+\left(d-d_{0}\right)^{2}} \\
& z_{1}=\sqrt{\left(a-a_{1}\right)^{2}+\left(b-b_{1}\right)^{2}+\left(c-c_{1}\right)^{2}+\left(d-d_{1}\right)^{2}} \\
& z_{2}=\sqrt{\left(a-a_{2}\right)^{2}+\left(b-b_{2}\right)^{2}+\left(c-c_{2}\right)^{2}+\left(d-d_{2}\right)^{2}} \\
& z_{3}=\sqrt{\left(a-a_{3}\right)^{2}+\left(b-b_{3}\right)^{2}+\left(c-c_{3}\right)^{2}+\left(d-d_{3}\right)^{2}}
\end{aligned}
$$

Figure 6. Expressions used in the location process of the neuronal networks based system.

$$
(x, y) \cong\left(x_{n}, y_{n}\right) \leftarrow \underline{n=z_{\text {_subscript }}} \rightarrow \min \left(z_{0}, z_{1}, z_{2}, z_{3}\right)
$$

This localization system can self-adapt when the environment changes. During the location process, when the system obtains the place more suitable for the detected signal, it saves automatically the RSS values from each AP in the row of that position.

Obviously, this adaptation system is efficient in those cases when the training map is big enough. If the database has only a few rows, the actualization of the values could fail into an error when it is making calculations.

\subsection{Triangulation Based System}

The triangulation method is another system used by sensors to self-locate in an environment. This system has been used by several positioning systems such as GPS. At the beginning, sensors must know the position where APs are placed and the gain of the antennas of the APs and of the sensors. Obtaining the received signal strength, we calculate the distance among the access points and the sensor that is measuring, and we want to locate, using expression 2 (defined for indoors environments by J. Lloret and others in [15]).

$$
d=10 \frac{P_{r \times 1 m}-\sum L_{i} \cdot P_{i}-P_{u}-P_{v}}{20}
$$

Where $\mathrm{P}_{\mathrm{rx} 1 \mathrm{~m}}$ is the AP received power at the distance of 1 meter, the sum of $\mathrm{L}_{\mathrm{i}} \cdot \mathrm{P}_{\mathrm{i}}$ are the propagation losses when the signal cross the wall. $\mathrm{P}_{\mathrm{u}}$ is the received power by the sensor and $\mathrm{P}_{\mathrm{v}}$ are the losses that have been studied in section 3 .

In order to achieve a good operation in this system, we need a minimum of three APs to be able to know the sensor position. First, we calculate the distances from the access points by means of received signal strength using expression 2. Once we known these distances (denoted as $d 1, d 2$ and $d 3$ ) and having all APs coordinates, we can calculate the position for the sensor using $\mathrm{X}$ and $\mathrm{Y}$ values shown in the equations 3 and 4.

$$
\begin{aligned}
& X=\frac{\left|\begin{array}{ll}
x 1^{2}+y 1^{2}-x 2^{2}-y 2^{2}-d 1^{2}+d 2^{2} & 2 \cdot(x 1-x 2) \\
x 3^{2}+y 3^{2}-x 2^{2}-y 2^{2}-d 3^{2}+d 2^{2} & 2 \cdot(x 3-x 2)
\end{array}\right|}{\left|\begin{array}{ll}
2 \cdot(x 1-x 2) & 2 \cdot(y 1-y 2) \\
2 \cdot(x 3-x 2) & 2 \cdot(y 3-y 2)
\end{array}\right|} \\
& Y=\frac{\left|\begin{array}{ll}
2 \cdot(x 1-x 2) & x 1^{2}+y 1^{2}-x 2^{2}-y 2^{2}-d 1^{2}+d 2^{2} \\
2 \cdot(x 3-x 2) & x 3^{2}+y 3^{2}-x 2^{2}-y 2^{2}-d 3^{2}+d 2^{2}
\end{array}\right|}{\left|\begin{array}{ll}
2 \cdot(x 1-x 2) & 2 \cdot(y 1-y 2) \\
2 \cdot(x 3-x 2) & 2 \cdot(y 3-y 2)
\end{array}\right|}
\end{aligned}
$$

The system bases its precision in the number of access points and in the Wireless channel characterization. An error taking measurements because of wall looses imply higher error estimating the position of the sensor. The way wall looses are measured are given by the approach shown in reference [15]. Higher number of APs gives higer precision to the system, but it makes the sensor calculate more distances (for every APs), so the sensor could need more processing time, diminishing the system performance. Many APs should imply higher responding time and more errors in the system.

\section{Performance Evaluation}

To test both systems using an autonomous process, we have developed a Java application. The application uses the map of the floor where APs and sensors are located. In both cases APs are distinguished using their MACs. In the Neuronal Network based system, we have trained the system using 30 sites as it is shown in figure 7. They are numbered in green and they are not in a square. When we started the system, the sensor just asked in which position it was placed taking into account data obtained in the training process and the signal variations introduced. We can also adapt the system by comparing obtained measures with real measures training again the system.

In the Triangulation based system, it is needed the position of every access point when the application starts. Distances from the sensors to each AP are calculated using expression 2 . The number of walls for each AP is estimated taking into account that the location of the sensor is given by the cross of their coverage circumferences (in this case, because there are 3 APs, there will be only one cross point for a given number of walls for each AP), as expressions 3 and 4 describe. 


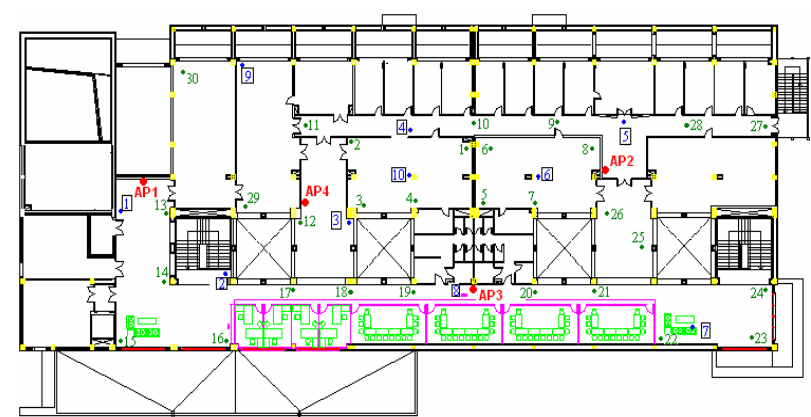

Figure 7. Floor and locations of the APs and sensors.

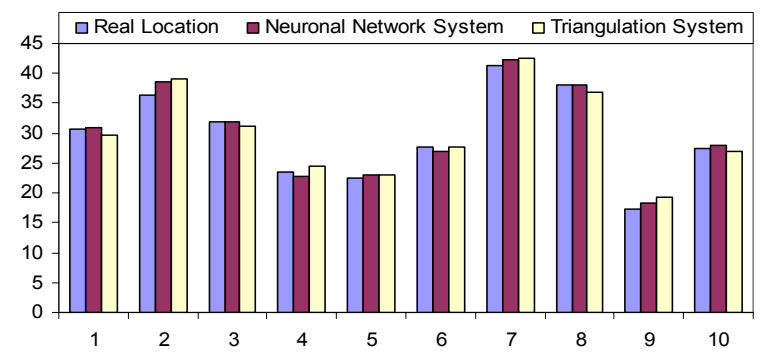

Figure 9. Systems comparison for $\mathrm{Y}$ axis.

We have tested both systems in the floor shown in figure 7. Measurements have been taken for 10 sites (all of them are inside a square in figure 7). Both systems and their comparison with real values are shown in figure 8 (for $\mathrm{X}$ axis) and figure 9 (for $\mathrm{Y}$ axis). The system that gives more accuracy values is the triangulation based system using the approach explained in reference [15], but we have checked that in an environment with different types of walls (different wall looses) it becomes extremely difficult to calculate the position, so, in these cases the best method is the Neuronal Network based system. The worst case locating a sensor using triangulation based system was site 4 with an error of 1,3 meters. The worst case locating a sensor using Neuronal Network based system was site 1 with an error of 2,5 meters. On the other hand, we estimate that using more training sites for the Neuronal Network based system, could give better results than the triangulation based system, without having the problem of wall looses.

\section{Conclusions}

This paper describes and discusses two Indoor Position Systems that are developed taking into account real measurements of signal variations. Triangulation based system has been the more accuracy system, but Neuronal Network based system could be better if there are different wall looses in the floor or if there are considered more sites, than the ones measured in this approach, to train the system.

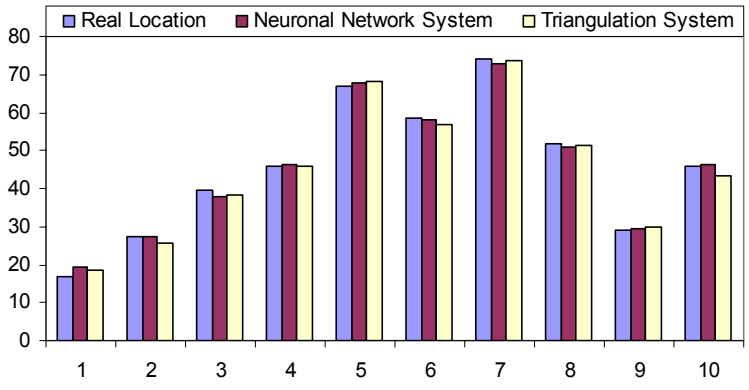

Figure 8. Systems comparison for $\mathrm{X}$ axis.

\section{References}

[1] P. Bahl and V.N. Padmanabhan. "Radar: An in-building user location and tracking system". Proceedings of the IEEE Infocom, Tel Aviv, Israel, Vol. 2, pp. 775-784, 2000.

[2] F.J. Gonzalez-Castaño and J.J. Garcia-Reinoso. "Bluetooth Location Networks". IEEE Globecom Int. Conference. Vol.1 pp. 233-237, 2002.

[3] L. M. Ni, Y. Liu, Y. C. Lau, and A. P. Patil. "Landmarc: Indoor location sensing using active RFID". Wireless Networks, 10(6), pp. 701-710, 2004.

[4] W. Park, M. Yoon. "The Implementation of Indoor Location System to Control ZigBee Home Network" SICEICASE. Int. Joint Conference pp. 2158 - 2161, 2006.

[5] G. Shreve and D. Kell. "A precision location network using Ultra Wide Band WLAN radios". In Proc. of Third IEEE Workshop on Wireless LANs, 2001.

[6] Andy Harter and Andy Hopper, "A Distributed Location System for the Active Office". IEEE Network, Vol. 8, No. 1, January 1994. pp. 62-70.

[7] Andy Hopper, Pete Steggles, Andy Ward, Paul Webster, "The Anatomy of a Context-Aware Application", Proc. of the $5^{\text {th }}$ ACM/IEEE Int. Conference on Mobile Computing and Networking, Seattle, Washington, USA, August 1999.

[8] N. B. Priyantha, A. Chakraborty and H. Balakrishnan, "The Cricket Location-Support System", Proc. 6th ACM MOBICOM, August 2000, pp. 32-43.

[9] P. Bahl andV. Padmanabhan, "RADAR: An In-Building RF-based User Location and Tracking System", IEEE INFOCOM, vol. 2, pp. 775-784. 2000

[10] Ekahau Positioning System, at: http://www.ekahau.com [11] Castro, P., Chiu, P., Kremenek, T., \& Muntz, R. "A Probabilistic Location Service for Wireless Network Environments". Ubiquitous Computing 2001. Sept. 2001.

[12] Herecast: WiFi Location-based Services/802.11 Positioning System. At http://www.herecast.com

[13] M Youssef, A Agrawala. "The Horus WLAN Location Determination System". At http://usenix.org

[14] M. Youssef, A. Agrawala, "Small-Scale Compensation for WLAN Location Determination Systems". Proc. of the 2003 ACM workshop on Wireless security, pp. 11-20. 2003.

[15] J. Lloret, J. J. Lopez, C. Turro and S. Flores, A Fast Design Model for Indoor Radio Coverage in the $2.4 \mathrm{GHz}$ Wireless LAN, $1^{\text {st }}$ Int. Symposium on Wireless Communication Systems, Port Louis (Mauritus), Sept. 2004. 